\title{
COOPERACIÓN UNIVERSITARIA INTERINSTITUCIONAL: UNA PROPUESTA DE DESARROLLO DE LA INVESTIGACIÓN EN ALIMENTOS EN CHILE
}

José Miguel Aguilera*

Fernando Vio** 


\section{RESUMEN}

Los alimentos y su importancia en la nutrición han alcanzado relevancia en el contexto nacional por su rol cada vez más evidente en salud pública y en las exportaciones no tradicionales. Este artículo pretende situar la problemática en el ambiente nacional y mundial y contribuir al debate público de cómo Chile debiera desarrollar en el corto y mediano plazo una estrategia coherente en el ámbito de la ciencia y tecnología que atienda los desafíos nacionales e internacionales que enfrenta el tema de alimentos.

\section{ABSTRACT}

Food and its importance for nutrition has reached an outstanding place in the national context due to the part it plays in public health and in non traditional exportations. This paper pretends to settle the issue in the national and world surrounding and, thus, contribute to the public debate as to how should Chile develop a coherent strategy in the scientific and technological environment in order for it to pay attention to the national and international challenges that the food subject has to face. 


\section{COOPERACIÓN UNIVERSITARIA \\ INTERINSTITUCIONAL: UNA PROPUESTA DE DESARROLLO DE LA INVESTIGACIÓN EN ALIMENTOS EN CHILE}

Introducción

Los alimentos han ido adquiriendo cada vez mayor importancia en la vida de las personas. Con el aumento de la expectativa de vida en la población, que en Chile es de 74 años en los hombres y 80 en las mujeres, la alimentación pasa a ser un elemento relevante en la calidad de vida, así como en la prevención de las enfermedades más frecuentes. Hoy día ya no nos enfermamos tanto de problemas infecciosos (diarreas, sarampión, difteria, polio y fiebre tifoidea en niños, tuberculosis en adultos), como en el pasado, sino de dolencias cardiovasculares, cánceres, diabetes, osteoporosis y otras, sobre las cuales se acrecienta la certeza científica de su estrecha relación con la dieta.

Evidencias acumuladas en los últimos años en salud pública y nutrición señalan que las enfermedades no dependen tanto de nuestra condición genética, sino de nuestra alimentación, actividad física, hábito de fumar, de las relaciones sociales e interpersonales, del enfrentamiento psicológico de la vida y del medio ambiente. De estos condicionantes, la alimentación es uno de los más importantes y la población lo ha percibido siendo cada vez más exigente frente a los alimentos que consume. Esto es evidente en los países desarrollados, que son altamente exigentes y que, curiosamente, se alimentan en buena medida de lo que producen países exportadores como el nuestro. 
En los últimos años el conocimiento en alimentos ha experimentado avances notables, que se reflejan en mejores productos disponibles en los mercados, no restringidos al sitio de origen sino, especialmente, a consumidores muy distantes. Este hecho permite que, en la actualidad, se pueda acceder a alimentos de alta calidad, con niveles de inocuidad muy altos y provenientes de lugares a los cuales los consumidores no tenían acceso sino hasta hace muy pocos años. Chile ha entrado muy fuerte en los mercados mundiales sin tener como respaldo la ciencia y tecnología que se requiere actualmente, existiendo un desfase entre lo que el país produce, con los ingresos que esto genera, y lo que se invierte en investigación en alimentos y nutrición.

La firma de nuevos tratados comerciales, y el consiguiente acceso a mercados muy sofisticados, trae como consecuencia una serie de exigencias a los productos que se exportan desde Chile y, por consiguiente, nuevos desafíos en ciencia y tecnología. Por ello, la generación de una base de conocimiento de alto nivel en alimentos es una necesidad fundamental para sostener, diversificar e incrementar la capacidad exportadora de nuestro país. Ello es y será fundamental en un medio muy competitivo, con elevados estándares de calidad. En el escenario de corto y mediano plazo, el rol que les cabe a las universidades en formación de recursos humanos y generación de conocimiento es fundamental, como se pretenderá demostrar.

De la granja a la mesa

Dado que los consumidores son cada vez más exigentes y conscientes de la importancia de una buena alimentación, se ha ido produciendo lentamente una verdadera "inversión" de la cadena alimentaria. Antes esta cadena iba de la granja a la mesa y hoy va desde el consumidor al productor, es decir, de la mesa a la granja. En otras palabras, son los consumidores y no los productores los que determinan qué se produce, cómo se produce y cómo se comercializa. Esto ha llevado a que la tendencia actual en alimentación y nutrición cambie radicalmente el centro de gravedad de la producción (oferta) hacia las demandas y derechos del consumidor a tener alimentos saludables y de alta calidad (Figura 1). 
Figura 1

Enfoque actual de la cadena alimentaria: de la mesa a la granja

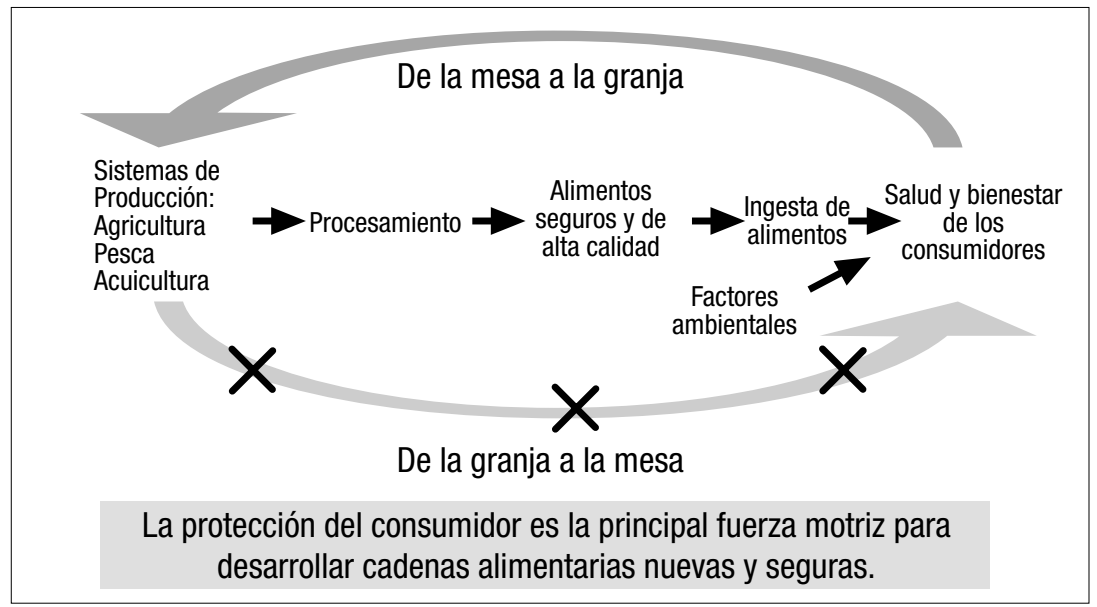

Sin embargo, la situación actual no favorece a los consumidores para que éstos demanden alimentos más saludables. Con los efectos de la globalización y urbanización creciente en todos los países, junto con los avances tecnológicos, aparece un actor muy importante que está en el medio de la cadena agroalimentaria: los supermercados. Ellos concentran la comercialización en grandes cadenas, controlando, por una parte, a los productores y, por otra, a los consumidores. Se trata de un fenómeno mundial que se da por igual en Europa, Estados Unidos y Japón, como en América Latina, África y Asia. En Chile, hace pocos años, el 50\% de los alimentos se comercializaba a través de supermercados y hoy superamos el $70 \%$.

Por otra parte, los consumidores están lejos de ser homogéneamente educados como para exigir los alimentos de calidad que requieren para una mejor vida y salud y, por lo tanto, su conocimiento de lo que se produce está aún lejano de ser una realidad. Los consumidores deberían seleccionar sus alimentos no sólo por su apariencia y sabor, sino por el contenido de nutrientes que contienen. Por ejemplo, al elegir una naranja, deberían considerar la cantidad de ácido ascórbico y jugo que contienen; al escoger una carne preocuparse no sólo de la dureza sino también de la cantidad de grasa que contiene $y$, al optar por una margarina, ver si tiene o no ácidos grasos transesterificados. 
Chile país exportador de alimentos

En las últimas décadas se ha ido consolidando en el país la vocación exportadora de productos agropecuarios, pesqueros y de alimentos procesados. Hoy las exportaciones de este sector representan cerca del 25\% del total, que en el año 2002 alcanzaron casi 20 mil millones de dólares. Más aun, esta actividad proporciona abundantes fuentes de empleo y acoge actividades de medianos y pequeños empresarios. El cobre, antiguamente llamado el "sueldo de Chile", hoy contribuye con menos del $40 \%$ del valor total exportado. Las exportaciones de productos del mar crecen a tasas de sobre el 10\% anual y las del sector agrícola al $14 \%$, lo cual indica que, en el corto y mediano plazo, existirá un gran dinamismo en el sector.

Vista en su conjunto, la actividad exportadora de alimentos hace aparecer a Chile como una "empresa" muy diversificada, donde actualmente once rubros son líderes mundiales en sus respectivos mercados de exportación. Sin embargo, esta situación no está libre de crítica: la mayoría de estas exportaciones corresponden a materias primas con bajo valor agregado como es el caso de filetes de pescado, uvas, kiwis, paltas y duraznos.

Valor agregado en los alimentos e innovación

Valor agregado implica, fundamentalmente, generar valor al consumidor de manera que esté dispuesto a pagar más por un beneficio adicional. Desde el punto de vista del productor, valor agregado es todo aquello que diferencia o mejora el producto, o hace más eficiente un proceso productivo. Puede ser tan sencillo como una mayor facilidad en el uso del producto, comodidad para almacenarlo o economía de tiempo. Por ejemplo, exportar cajas de frutas mixtas para consumo familiar podría ser una forma simple de dar valor agregado a estas exportaciones.

Innovación, por su parte, es la actividad sistemática por la cual se persigue agregar valor al producto. Ya a principios de siglo los economistas propusieron que el motor del crecimiento económico y de la prosperidad material era la introducción de nuevas ideas. Las 
innovaciones exitosas desplazan a tecnologías inferiores mediante lo que se denomina el proceso de creación destructiva; a través de la imitación, adaptación y difusión, estas innovaciones se extienden al sistema económico. Economistas posteriores han identificado que el cambio tecnológico, dentro del cual la innovación juega un rol fundamental, es la única razón para explicar el crecimiento sostenido de los estándares de vida en países más desarrollados. El cambio tecnológico es, a su vez, una consecuencia de los descubrimientos científicos o, en otras palabras, el conocimiento es un bien económico (Montes, 2002).

El problema reside en que el empresariado nacional parece no considerar al progreso tecnológico entre los factores importantes para el desarrollo económico. Al ser consultados sobre medidas que favorecerían una reactivación económica, líderes del sector apuntaron fundamentalmente a medidas externas a las empresas: alza del tipo de cambio, alternativas de financiamiento para PYMES, estabilidad en las normas laborales, manutención de "reglas del juego claras", modernización del Estado y menor burocracia, y una eliminación de las distorsiones microeconómicas. Esto contrasta con opiniones de expertos extranjeros como Jeffrey Sachs, profesor de Harvard, quien, al ser consultado sobre un cambio en la estructura de las exportaciones nacionales, manifestó: "Es alarmante el bajo nivel de tecnología de Chile".

Visión estratégica del área alimentos

Una pregunta todavía sin respuesta es como enfrentará Chile las oportunidades que emergen de los tratados comerciales recientemente firmados. Entrar decididamente en el negocio de la alimentación en el ámbito mundial obliga a tener una visión estratégica de largo plazo similar a la de los competidores. Entre éstos se cuentan otros países del hemisferio sur con potencial exportador en el rubro agroalimentario como Australia, Nueva Zelandia y Sudáfrica, y también algunas grandes transnacionales de la industria alimentaria. En ambos casos se trata de competidores que creen en la ciencia y la tecnología como un instrumento decisivo para el desarrollo y sustentabilidad de sus exportaciones o negocios, respectivamente. 
Entre otros indicadores, y en relación con Chile, los países citados anteriormente invierten una mayor proporción del Producto Interno Bruto (PIB) en Investigación y Desarrollo (I\&D), tienen más doctores e ingenieros involucrados en estas actividades y la empresa privada juega un papel fundamental en la innovación y el crecimiento (Montes, 2002). En el caso particular de Nueva Zelandia, que también se caracteriza por ser un país exportador de recursos naturales y que cuenta con una posición geográfica similar a Chile, el gobierno ha decidido ingresar al grupo de los primeros 15 miembros de la Organización para la Cooperación y Desarrollo Económicos (OCDE) en términos de ingreso per capita. La forma de lograrlo es mediante el fomento de la innovación, el fortalecimiento de la base macroeconómica, la mejora de su sistema nacional de innovación, el desarrollo de aptitudes y talentos de sus habitantes y la focalización de recursos gubernamentales en ciertas áreas estratégicas (Anónimo, 2002).

Las multinacionales de la alimentación mantienen actividades de I\&D que van desde ciencia básica relacionada con sus competencias centrales hasta centros de desarrollo de productos cada vez más cercanos a los consumidores de los diversos países donde operan. Por ejemplo, Unilever (que también tiene negocios en detergentes y productos de aseo personal) cuenta con 9.000 empleados trabajando en I\&D alrededor del mundo e invierte anualmente alrededor del $2 \%$ de las ventas, que equivalen a unos US $\$ 750$ millones. Para esta compañía, la I\&D "debe proveer de las ventajas competitivas en los distintos negocios que le permitan alcanzar sus metas de crecimiento".

Integración de la empresa privada en la investigación

En ejemplos recientes de desarrollo tecnológico acelerado de países pequeños como el nuestro, la empresa privada juega un rol fundamental. Al fin y al cabo de lo que se trata es de exportar alimentos con mayor valor agregado y éstos salen de las empresas. Las estadísticas actuales muestran que, en Chile, la mayor parte de la investigación se hace en las universidades y los institutos dependientes del Estado y sólo una proporción menor en las empresas (Véase Fig. 2). Esto contrasta con lo que ocurre en países donde la tecnología es motor importante del desarrollo, en cuyo caso sobre el $40 \%$ de la I\&D se 
realiza en las empresas. En el corto plazo resulta difícil prever que esta situación pueda ser revertida pero, a la luz de la aparición de nuevas fuentes de financiamiento para la I\&D nacionales, se hace necesario revisar el modelo actual.

Movilizar a las empresas hacia las universidades e incentivar la I\&D dentro de las empresas no es sino adecuarse a sistemas probadamente exitosos en economías emergentes basadas en el conocimiento. Dado que en la producción de alimentos y procesamiento de recursos naturales pueden participar de manera importante las pequeñas y medianas empresas (PYMES), su acercamiento a la investigación tecnológica en universidades asegura una mayor equidad en la inversión en ciencia y tecnología.

Figura 2

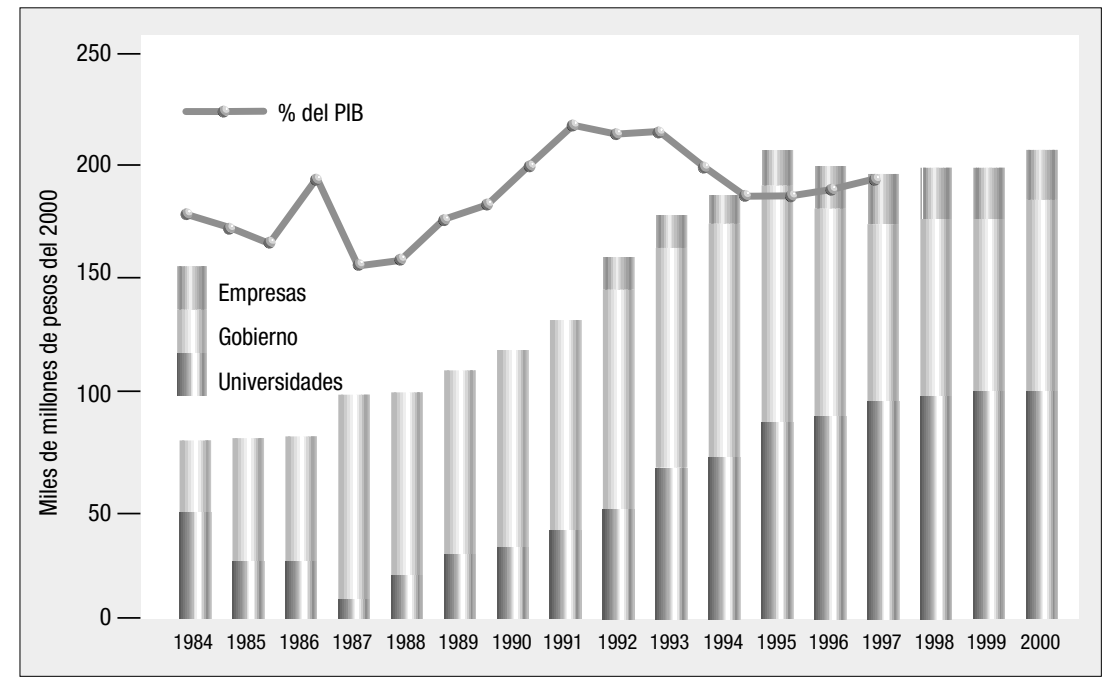

El Programa Marco de la Comunidad Europea

A modo de ejemplo de lo anterior, conviene revisar las acciones de I\&D emprendidas por la Comunidad Europea. Se ha definido el Espacio Europeo de la Investigación como un proyecto de futuro que fomente la calidad científica, la competitividad y la innovación, mediante una mejor cooperación y coordinación entre los interesados, en los distintos niveles. 
Dentro del $6^{\circ}$ Programa Marco de I\&D 2002-2006, nuestros socios europeos han definido siete áreas prioritarias, dentro de todo el espectro posible en Ciencia y Tecnología (C\&T), una de las cuales se denomina Seguridad Alimentaria y Riesgos para la Salud. La formación de recursos humanos y el reforzamiento de la infraestructura de investigación tienen las mayores asignaciones presupuestarias, concordante con el esfuerzo por formar una fuerte base de apoyo para actividades productivas.

Otro aspecto novedoso del enfoque es que favorece la formación de grandes consorcios de investigación con participación de agencias de gobierno, universidades y sector privado, que enfrenten importantes problemas de manera multidisciplinaria. Esto, por una parte, reconoce que el expertise se encuentra distribuido (y es bueno que así sea) y, por otra, que la visión de una cadena alimentaria obliga a coordinar esfuerzos de diferentes disciplinas para la resolución de problemas complejos.

Formación de recursos humanos y de una mejor infraestructura para la investigación

Competir en las "ligas mayores" de la alimentación y nutrición mundial requiere cumplir con estándares básicos de infraestructura humana y material. Hasta hace algunos años, el total de doctores graduados anualmente en las universidades nacionales no superaba los setenta. Gracias a esfuerzos importantes de la Comisión Nacional de Investigación Científica y Tecnológica (CONICYT), hoy existe en el país un número apreciable de programas de doctorado en $C \& T$ y el número de graduados ha aumentado significativamente. Aunque esto es encomiable desde el punto de vista cuantitativo, aún existen áreas en que el país no logra formar especialistas de alto nivel, necesarios para su desarrollo.

Actualmente existen, entre otras, carencias importantes en especialistas en microbiología y toxicología de alimentos, siendo ambas disciplinas fundamentales para asegurar la inocuidad de los alimentos que exportamos. Algo similar ocurre en el área de tecnologías emergentes para la preservación de alimentos y el aseguramiento de la calidad. La renovación y puesta al día del equipamiento viene normalmente asociada a proyectos específicos y no con el criterio de 
fortalecer centros de excelencia que sirvan en algún momento para respaldar, con los estándares más altos posibles, las demandas de mercados foráneos. El hecho de que nuestros productos de exportación van a ser examinados cada vez más acuciosamente por las agencias reguladoras extranjeras, debiera hacernos anticipar episodios lamentables y revisar la capacidad de respuesta científica que poseemos.

Al respecto, las universidades debieran asociarse para ofrecer programas de posgrado que cubran las falencias expresadas y especializarse en metodologías analíticas que aseguren que, como país, estamos cubiertos frente a las eventualidades anteriormente expuestas.

Coordinación y cooperación entre los actores de la I\&D.

Un cambio de enfoque hacia una visión integrada de la actividad agroalimentaria en el ámbito nacional, que responda a las demandas de los consumidores y que provea de mayores recursos humanos y materiales para la innovación, es una condición necesaria pero no suficiente para garantizar un impacto en la competitividad. Es necesario además que los recursos, siempre escasos, se acoplen y potencien en torno a resolver problemas reales, anticipándose a las amenazas propias de la actividad que aparecen diariamente, como son, por ejemplo, nuevas variedades de plagas, microorganismos emergentes y otras. En este sentido, es importante que Chile de al mundo una imagen de país serio y confiable en lo que a C\&T se refiere. Esto significa asegurar que se han tomado todas las medidas durante la producción, procesamiento, almacenaje, distribución y flete de los productos alimentarios, de modo que éstos no representen un riesgo apreciable para la salud de los consumidores.

Entre los muchos competidores del Tercer Mundo que encuentran bajas barreras de entradas a los mercados de exportación de alimentos, nuestro país debiera posicionarse prontamente con una política de Estado respecto a esta actividad exportadora y crear una Agencia Agroalimentaria que coordine las ahora dispersas acciones del Estado para el sector. Un país no puede participar en el comercio internacional si no establece una reputación de buena regulación. Es indispensable que el sector privado exportador se sienta respaldado por acciones en $C \& T$ que promuevan aumentos de la productividad, mejoramiento de la calidad e inocuidad de los productos, y la sustentabilidad de los procesos. 
Coordinación de los esfuerzos del Estado

Para enfrentar los desafíos de los mercados de exportación y de la problemática nutricional, es necesario coordinar esfuerzos y presentar amplios frentes comunes. La tendencia mundial es hacia una centralización de la regulación para los alimentos, mientras actualmente en Chile el marco regulatorio depende de diferentes instituciones. Por una parte, están las normas establecidas desde la División del Ambiente y Unidad de Nutrición del Ministerio de Salud y el control sanitario de los alimentos realizado por el Servicio de Salud Metropolitano del Ambiente (SESMA), y los Servicios del Ambiente de los 26 Servicios de Salud a lo largo del país, apoyados por los laboratorios del Instituto de Salud Pública (ISP). Además están la Oficina de Planificación Agrícola (ODEPA), que emite normas sobre productos alimentarios, y el Servicio Agrícola y Ganadero (SAG), que controla los alimentos dependientes del Ministerio de Agricultura; también el Servicio Nacional de Pesca (SERNAPESCA) y el Instituto de Normalización (INN), que dependen del Ministerio de Economía y ejercen funciones normativas y de control en el tema de alimentos. Existe escasa, por no decir ninguna, coordinación entre estas instituciones que, muchas veces, se superponen en sus funciones, lo que crea desconcierto en los productores y distribuidores de alimentos y una pobre imagen internacional.

Para enfrentar este tema, se ha planteado la creación de una Agencia Agroalimentaria que coordine la supervisión de la calidad e inocuidad de los alimentos, desde el inicio de la cadena productiva hasta su consumo y exportación. Debería ser una entidad autónoma, que pueda contratar estudios y análisis, generar normas y reglamentos con base científica, incorporando la opinión y participación de actores no gubernamentales, como la empresa privada y la academia. Además, debería cubrir todo el territorio nacional, utilizando los recursos humanos y materiales existentes, dispersos actualmente en diferentes instituciones. Entre sus funciones más importantes estaría el mantener información actualizada del tema, con datos y estudios de los organismos públicos y privados, la cual debería estar disponible en un sitio web; generar información epidemiológica científicamente validada por un Comité de Expertos; acreditar laboratorios de análisis de acuerdo a normas internacionales; fomentar la incorporación de tecnología moderna -por ejemplo, $\mathrm{PCR}^{1}$ en tiempo real- en los 
laboratorios nacionales con actualización continua de métodos e instrumentación.

Para ello debería contar con el presupuesto necesario que permita, además, disponer de un fondo competitivo para investigación y estudios. Con ello se trataría de cambiar el actual sistema de control, que se ejerce al final de la cadena, por uno preventivo basado en la responsabilidad de las empresas, las que para ello deberán implementar (o reforzar) el Análisis de Riesgo y Control de Puntos Críticos en los procesos productivos. Por otra parte, se cambiaría el enfoque reactivo frente a problemas por uno que anticipara posibles focos de controversia respecto a calidad y seguridad.

Cooperación interinstitucional para un enfoque multidisciplinario del tema de alimentos: Proyecto Alimentos Unión Europea

Una vez firmado el Acuerdo de Cooperación Científica y Tecnológica entre Chile y la Unión Europea (UE) se definieron temas de interés conjunto, quedando como una de las prioridades el tema de alimentos. Ante ello, CONICYT formó un grupo de trabajo con representación de todas las universidades del país en septiembre de 2002. Este grupo solicitó expresiones de interés a todos los investigadores chilenos relacionados con el tema, recibiendo 77 respuestas. El grupo de trabajo las analizó y agrupó por subtemas que estuviesen de acuerdo con lo solicitado por la UE en su llamado al $6^{\circ}$ Programa Marco (2002-2006). Además, se realizó una reunión con el encargado del tema de alimentación en la UE, quien visitó Chile en el año 2002. En noviembre de ese mismo año, un representante del grupo viajó a Bruselas en la delegación chilena que asistió al lanzamiento del $6^{\circ}$ Programa Marco. En enero de 2003 tuvo lugar un seminario en Santiago con los máximos representantes en Ciencia y Tecnología de la UE y abierto a toda la comunidad científica relacionada con las temáticas definidas dentro de dicho programa.

Mientras tanto, los investigadores iniciaron contacto con consorcios europeos que habían presentado proyectos al $6^{\circ}$ Programa Marco, incorporándose muchos de ellos, como parte de dichos

1 Polymerasa Chain Reaction. 
consorcios, en temas como acrilamida, probióticos, envases, contaminación avícola y trazabilidad, estrategia que dio como resultado la aprobación de dos proyectos. Pero, de inmediato, apareció la necesidad de hacer un proyecto país en el tema de alimentos que exprese nuestras necesidades en ciencia y tecnología. Para ello, se reunió en junio de 2003 un grupo de investigadores que elaboró un proyecto presentado a la UE para su financiamiento, a través del Comisario para la Ciencia y Tecnología de la UE, Philippe Busquin, quien visitó Chile en julio de 2003. Durante la visita del alto funcionario, se le consultó en qué áreas de investigación tendría Chile más oportunidades en los programas de la UE, respondiendo que en seguridad y calidad alimentaria. Este proyecto tiene como objetivo general implementar sistemas de innovación tecnológica para mejorar la calidad e inocuidad de los alimentos en beneficio de la salud y calidad de vida de los consumidores, con los siguientes objetivos específicos:

- Asegurar al consumidor la calidad e inocuidad de los alimentos y fortalecer la capacidad exportadora nacional.

- Implementar tecnologías innovadoras en el área de la calidad e inocuidad de los alimentos.

- Formar recursos humanos en las nuevas tecnologías.

- Establecer un modelo para otros países exportadores de la región latinoamericana.

El proyecto abarca tres áreas prioritarias: frutas y hortalizas, carnes y vinos. Para enfrentar desafíos en estas áreas y cumplir con los objetivos generales, la calidad e inocuidad de los productos alimentarios fueron definidos como los dos grandes temas transversales en los que se deben incorporar innovación tecnológica para alcanzar los estándares exigibles por los países con los que se han firmado acuerdos económicos. Como ejes que subyacen en todo lo anterior, existen dos temas generales: el desarrollo y aplicación de nuevas tecnologías en la cadena agroalimentaria y la formación de recursos humanos en alimentos.

El resultado esperado de este proyecto es que se incorporen tecnologías innovadoras y se preparen recursos humanos que sean capaces de mejorar la calidad e inocuidad de los alimentos producidos en Chile. Esta meta irá en beneficio directo de la economía exportadora del país y de los consumidores, tanto nacionales como extranjeros. 


\section{REFERENCIAS BiBLIOGRÁFICAS}

Office of the Prime Minister of New Zealand, "Anónimo 2002", en Growing and Innovative, New Zealand, Febrero, 2002.

Montes, MA, Chile: Innovación como motor del crecimiento económico, Memoria para optar al título de Ingeniero Civil de Industrias, Pontificia Universidad Católica de Chile, Santiago, Chile, 2002.

WHO, Diet, Nutrition and the prevention of chronic diseases, Report of a joint WHO/FAO Expert Consultation, (WHO Technical Report Series $\mathrm{n}^{\circ}$ 916), Geneva, World Health Organization, 2003. 